\title{
Limitations of cardiothoracic ratio derived from chest radiographs to predict real heart size: comparison with magnetic resonance imaging
}

Paulius Simkus ${ }^{1}$, Manuel Gutierrez Gimeno ${ }^{1}$, Audra Banisauskaite ${ }^{1,2}$, Jurate Noreikaite ${ }^{1}$, David McCreavy ${ }^{1}$, Diana Penha ${ }^{1}$ and Monika Arzanauskaite ${ }^{1,3^{*}}$ (1)

\begin{abstract}
Background: Cardiothoracic ratio (CTR) in chest radiographs is still widely used to estimate cardiac size despite the advent of newer imaging techniques. We hypothesise that a universal CTR cut-off value of $50 \%$ is a poor indicator of cardiac enlargement. Our aim was to compare CTR with volumetric and functional parameters derived from cardiac magnetic resonance imaging (MRI).
\end{abstract}

Methods: 309 patients with a chest radiograph and cardiac MRI acquired within a month were reviewed to assess how CTR correlates with multiple cardiac MRI variables: bi-ventricular EDV (absolute and indexed to body surface area), EF, indexed total heart volume and bi-atrial areas. In addition, we have also determined CTR accuracy by creating multiple ROC curves with the described variables.

Results: All cardiac MRI variables correlate weakly but statistically significantly with CTR. This weak correlation is explained by a substantial overlap of cardiac MRI parameters in patients with normal and increased CTR. For all variables, CTR was only mildly to moderately better than a chance to discriminate cardiac enlargement (AUC 0.6-0.7). Large CTR values (>55\%) are specific but not sensitive, while low CTR values $(<45 \%)$ are sensitive but not specific. Values in between are not sensitive nor specific.

Conclusions: CTR correlates weakly with true chamber size assessed by gold standard cardiac MRI and has a weak discriminatory power. Thus, clinical decisions based on intermediate CTRs (45-55\%) should be avoided. Large CTRs (>55\%) are likely indicative of true heart chamber enlargement. Low CTRs $(<45 \%)$ are likely indicative of normal heart size.

Keywords: Cardiothoracic ratio, Cardiac magnetic resonance imaging, Chest radiograph, Cardiac imaging

*Correspondence: Monika.Arzanauskaite@lhch.nhs.uk

1 Radiology Department, Liverpool Heart and Chest Hospital, Thomas Drive, Liverpool L14 3PE, UK

${ }^{3}$ Cardiovascular Research Center_ICCC, Hospital de La Santa Creu I Sant Pau, IIB-Sant Pau, Barcelona, Spain

Full list of author information is available at the end of the article

\section{Key Points}

- CXR-derived CTR correlates weakly with cardiac chamber enlargement detected on cardiac MRI.

- No single CTR cut-off value has good accuracy in diagnosing cardiomegaly.

- The cut-off value of CTR $>50 \%$ has limited sensitivity and specificity. 
- Intermediate CTR values (45-55\%) do not reliably indicate heart size.

\section{Background}

Chest radiographs (CXR) are routinely used to assess the lungs and mediastinum. Cardiothoracic ratio (CTR) is a simple method to evaluate the heart size on chest radiographs. Despite having been introduced more than 100 years ago [1], it is still commonly reported nowadays, even though new imaging techniques have been developed. This is probably because CTR can be easily measured and simply interpreted on a widely available and inexpensive imaging study. Nevertheless, CTR depends on multiple technical and anatomic factors that can contribute to an inaccurate assessment of the real heart size. CTR is only considered reliable if calculated from a frontal upright postero-anterior (PA) chest radiograph. The supine position and any other antero-posterior (AP) projection overestimate cardiac size due to a magnification effect caused by the heart being closer to the imaging cassette. In addition, there are other cardiac and noncardiac aspects such as sub-optimal inspiratory effort, patient's or thoracic cage abnormalities that could influence the value of CTR [2-4].

CTR is defined as the ratio between the maximal horizontal cardiac diameter and the maximal horizontal inner thoracic cage diameter [2]. A CTR $>0.5$ (or $>50 \%$ ) is considered abnormal. In radiology reports, terms like "cardiomegaly" or "increased heart size" are commonly used to describe an increased CTR. Despite its broad use, some researchers have questioned the real value of this arbitrary cut-off point. For example, in a study with patients undergoing coronary angiography due to angina, a CTR between 42 and $49 \%$ was associated with a higher risk of all-cause mortality or major coronary event (death, nonfatal myocardial infarction) compared to patients with CTR $<42 \%$ [5].

In modern diagnostic imaging, newer modalities allow to thoroughly examine the three-dimensional structure of the heart, assessing chamber size and function. Some studies have questioned the correlation between CTR and cardiac parameters derived from other imaging techniques with controversial results. In comparisons done within specific cardiac pathologies, there is usually an absence of correlation with CTR or CTR correlates with the size of only one or two cardiac chambers [6, 7]. A systematic review by Loomba et al. showed that CTR is sensitive for identifying an increased left ventricular volume on echocardiography (sensitivity $83.3 \%$ ) but lacks specificity (45.4\%) [8]. Larger CTRs were related to increased mortality risk in adults with congenital heart disease [6]. Apart from chest radiographs, CTR can also be measured using other imaging modalities like CT and MRI. However, Schlett et al. found that CTR derived from CT scout images did not correlate to LV parameters measured from the same CT scan [9].

Cardiac MRI is a modern, high-resolution, ionising radiation-free technique that is now considered the gold standard for cardiac volumetry and function assessment [10-12]. We hypothesise that the cut off value of $\mathrm{CTR}=50 \%$ is a poor indicator of cardiac enlargement and should be interpreted with caution. The primary aim of this study was to compare the CTR to indexed cardiac volumetry values. Secondary objectives were: (1) to evaluate the isolated correlation between CTR and individual cardiac chamber measurements, (2) to stratify the results by age and gender using the dedicated normal cardiac MRI values for comparison.

\section{Methods}

\section{Study population}

Institutional Review Board approval was waived by our Research and Innovation Department, which classified the study as Service Evaluation. A retrospective single centre study was performed. A random list of 532 chest radiographs performed between 2016 and 2020 was selected with the following inclusion criteria: (1) adults ( $>18$ years old) with a chest radiograph and (2) cardiac MRI performed in a time period of less than one month. The initial list was reduced to 438 patients after excluding duplicate entries (patients with more than one radiograph) and excluding patients with AP chest radiographs. Only PA chest radiographs were considered for analysis. Additionally, cases with obscured heart contours due to pleural effusion or pulmonary consolidation were excluded. Regarding cardiac MRI, patients were excluded if the scan was incomplete (i.e. no ventricular functional module acquired) or if the scan quality was severely affected by artefacts resulting in non-analysable datasets. Once the exclusion criteria were applied, the final sample was formed by 309 patients. Figure 1 shows the key components of the study design.

\section{Chest radiographs}

Two radiologists (J.N., P.S.) measured the CTR dividing the largest horizontal heart diameter by the largest horizontal internal thoracic cage diameter (Fig. 2) using a DICOM viewer software package (Carestream VUE 12.2.2.2, Carestream Health Inc).

\section{Cardiac MRI}

Studies were performed on 1.5-T and 3-T cardiac MRI systems (Aera and Vida, Siemens Medical Solutions; Achieva, Philips Healthcare) with breath-holding and electrocardiography (ECG)-triggering techniques. All 


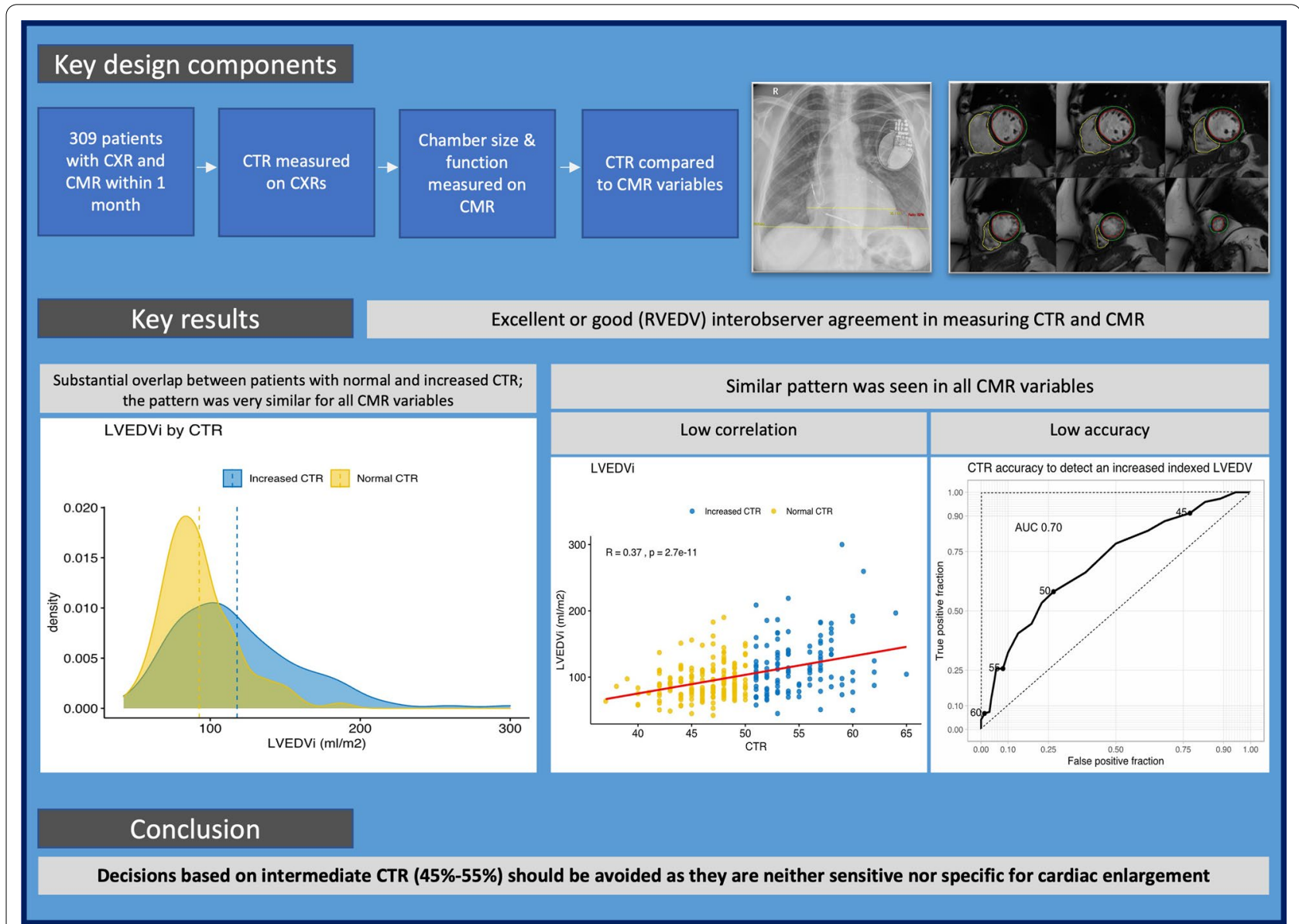

Fig. 1 Key components of study design and results

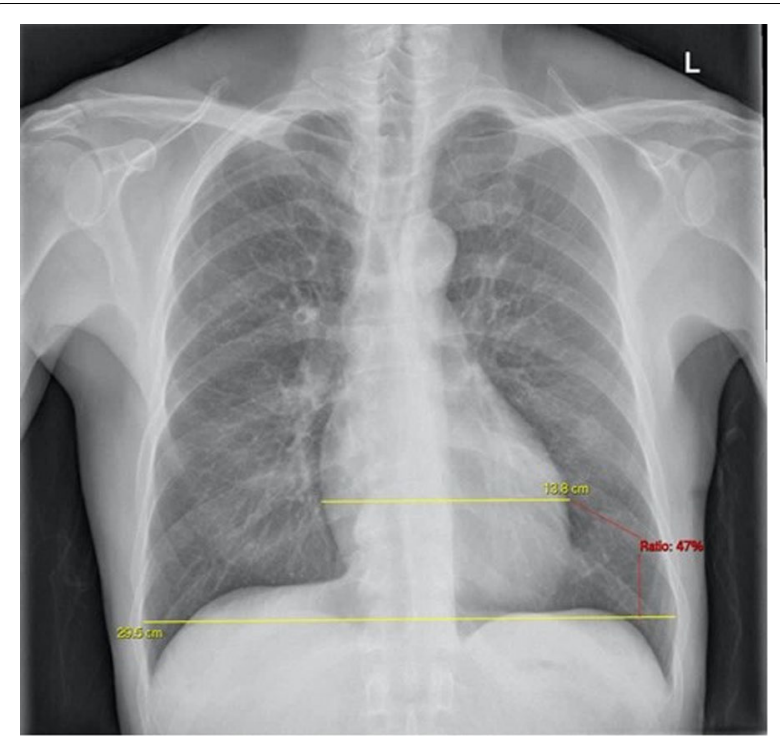

Fig. 2 The cardiothoracic ratio on PA chest radiograph. The maximum transverse cardiac diameter is divided by the maximum transverse diameter of the thorax and multiplied by 100 analysed studies had a ventricular functional module available (horizontal long axis, vertical long axis and LVOT views, and a short axis stack from base to apex).

Cardiac MRI scans were analysed using dedicated postprocessing software $\left(\mathrm{CVI}^{42}\right.$ Version 5.11.4, Circle Cardiovascular Imaging Inc) by two radiologists with 2- and 3-years of experience in cardiac MRI analysis (PS, MG), with the senior review by a Level 3 certified cardiac MRI practitioner (M.A.) where necessary.

The following protocol was performed: firstly, automated left and right ventricular volume analyses were obtained. Then, the software traced biventricular endocardial and LV epicardial borders in end-diastole and end-systole to obtain the end-diastolic volumes (EDV), end-systolic volumes (ESV), bi-ventricular ejection fraction (EF) and LV mass. A manual correction was applied where needed. Papillary muscles were included in the blood pool when measuring ventricular volumes and excluded from the blood pool when calculating LV mass to increase the accuracy and align with the methods of the normal reference studies [15]. The end-systolic 
atrial areas and volumes were also obtained using horizontal and vertical long-axis cine images. All obtained values were indexed to the patient's body surface area, calculated using the Mosteller formula. Additionally, the indexed total heart volume (THVi) was calculated by adding LVEDVi, RVEDVi, indexed LA volume and indexed RA volume.

After performing the measurements, studies were classified into nine different diagnostic categories: ischaemic heart disease, non-ischaemic cardiomyopathy, myocarditis/pericarditis, heart failure/LV dysfunction, valvulopathy, congenital heart disease, $\geq 2$ pathologies, normal/ screening and other diseases. The recorded diagnoses were obtained based on the original report and combined with the researchers' observations. In cases of discrepancy between the researchers' interpretation and the reported diagnosis, a consensus was reached by reviewing the studies in a team with a Level 3 certified cardiac MRI practitioner (M.A.).

\section{Interobserver variability}

Interobserver variability was assessed by a second analysis of approximately $10 \%$ of the total sample: 30 CXR and 30 cardiac MRI studies were randomly selected.

\section{Data analysis}

Statistical analysis was performed using SPSS (version 25.0). First, normality was evaluated using the ShapiroWilk test. According to the results, the following statistics were applied: Spearman correlation ranks to assess correlations, Mann-Whitney $U$ test for comparison between groups and the Intraclass correlation coefficient (ICC) for interobserver variability (absolute agreement estimated using a two-way mixed effect model). Statistically significant differences were defined by a $p$-value less than 0.05. Graphics and Receiver operating characteristic (ROC) analysis were obtained using $\mathrm{R}$ and its library plotROC $[13,14]$.

Categorical data are expressed as counts and percentages. Continuous variables are presented as mean \pm standard deviation or as median with inter-quartile range.

\section{Results}

\section{Study population and baseline characteristics}

Among 309 total individuals, 215 (69.6\%) were males and $94(30.4 \%)$ females. The average patient's age was $57.9 \pm 14.8$ years. There was no significant age difference between males and females ( $58 \pm 16$ vs. $58 \pm 15$ years, respectively; $p>0.05$ ). The average time interval between cardiac MRI and chest radiographs was $9.08 \pm 4.95$ days.

Cardiac MRI diagnoses and their distribution are summarised in Table 1. Ischaemic heart disease was the
Table 1 Distribution of diagnoses and their characteristics

\begin{tabular}{lccr}
\hline Pathology & Cases (\%) & $\begin{array}{l}\text { Normal: } \\
\text { increased } \\
\text { CTR }\end{array}$ & क: $^{+}$ \\
\hline Ischaemic heart disease & $114(36.9)$ & $77: 37$ & $25: 89$ \\
Non-ischaemic cardiomyopathy & $67(21.7)$ & $32: 35$ & $23: 44$ \\
Myocarditis/pericarditis & $22(7.1)$ & $14: 8$ & $7: 15$ \\
Heart failure/LV dysfunction & $12(3.9)$ & $9: 3$ & $4: 8$ \\
Valvulopathy & $22(7.1)$ & $13: 9$ & $12: 10$ \\
Congenital heart disease & $6(1.9)$ & $1: 5$ & $1: 5$ \\
$\geq 2$ pathologies & $21(6.8)$ & $14: 7$ & $4: 17$ \\
Normal/screening & $25(8.1)$ & $22: 3$ & $12: 13$ \\
Other & $20(6.8)$ & $12: 8$ & $6: 14$ \\
\hline
\end{tabular}

Table 2 Interobserver agreement demonstrated as intraclass correlation coefficient (ICC) for measuring CTR and cardiac MRI parameters

\begin{tabular}{ll}
\hline Parameter & ICC between observers \\
\hline CTR & $0.937(0.869-0.97)$ \\
LVEDV & $0.985(0.962-0.994)$ \\
LVESV & $0.992(0.942-0.997)$ \\
LVEF & $0.977(0.936-0.99)$ \\
LV mass & $0.984(0.965-0.993)$ \\
RVEDV & $0.846(0.675-0.927)$ \\
RVESV & $0.975(0.919-0.99)$ \\
RVEF & $0.935(0.856-0.97)$ \\
LA volume & $0.977(0.948-0.99)$ \\
LA area & $0.959(0.914-0.98)$ \\
RA volume & $0.926(0.845-0.965)$ \\
RA area & $0.91(0.811-0.957)$ \\
\hline
\end{tabular}

Values in parentheses are $95 \%$ confidence intervals

ICC intraclass correlation coefficient, CTR cardiothoracic ratio, MRI magnetic resonance imaging, LVEDV left ventricular end-diastolic volume, LVESV left ventricular end-systolic volume, $L V E F$ left ventricular ejection fraction, $L V$ left ventricle, RVEDV right ventricular end-diastolic volume, RVESV right ventricular end-systolic volume, $R V E F$ right ventricular ejection fraction, $L A$ left atrium, $R A$ right atrium

most common pathology, with 114 cases (36.9\%). 25 (8.1\%) studies were normal. Cases with diagnoses that were less common and could not be assigned to any of the pre-defined main groups were included in "Other".

\section{Reproducibility of CTR and cardiac MRI measurements} There was an excellent interobserver agreement in CTR measurements (ICC estimate 0.937; 95\% CI 0.8690.97). Interobserver agreement was also excellent in all the analysed cardiac MRI parameters (ICC $>0.9$ ) except in the RVEDV, where it was good (ICC 0.846), Table 2. 
Table $3 \mathrm{MRI}$ parameters between normal vs increased CTR and CTR correlation with cardiac MRI parameters

\begin{tabular}{lccc}
\hline MRI parameter & CTR $\leq \mathbf{5 0}(\mathbf{N}=\mathbf{1 9 4})$ & CTR $>\mathbf{5 0}(\mathbf{N}=\mathbf{1 1 5})$ & CMR and CTR correlation \\
\hline LVEDVi $\left(\mathrm{ml} / \mathrm{m}^{2}\right)$ & $89(76-106)$ & $110(86-139)$ & $0.367(p<0.005)$ \\
LVEF $(\%)$ & $54(42.5-61)$ & $42(29-55)$ & $-0.341(p<0.005)$ \\
Indexed LV mass $\left(\mathrm{mg} / \mathrm{m}^{2}\right)$ & $68(60-78.5)$ & $80(66-98)$ & $0.319(p<0.005)$ \\
RVEDVi $\left(\mathrm{ml} / \mathrm{m}^{2}\right)$ & $75(64-89)$ & $84(66-102)$ & $0.170(p<0.005)$ \\
RVEF $(\%)$ & $58(53-63)$ & $54(41-59)$ & $-0.241(p<0.005)$ \\
Indexed LA volume $\left(\mathrm{ml}^{2} / \mathrm{m}^{2}\right)$ & $40(32-50)$ & $56(40-75)$ & $0.389(p<0.005)$ \\
Indexed LA area $\left(\mathrm{cm}^{2} / \mathrm{m}^{2}\right)$ & $13(10-15)$ & $15(12-18)$ & $0.360(p<0.005)$ \\
Indexed RA volume $\left(\mathrm{ml}^{2} / \mathrm{m}^{2}\right)$ & $33.5(27-42.5)$ & $40(31-58)$ & $0.247(p<0.005)$ \\
Indexed RA area $\left(\mathrm{cm}^{2} / \mathrm{m}^{2}\right)$ & $11(9-12)$ & $12(10-15)$ & $0.249(p<0.005)$ \\
\hline
\end{tabular}

Values in the two middle columns are median (interquartile range), and values in the far-right column are correlation coefficients

$M R I$ magnetic resonance imaging, CTR cardiothoracic ratio, $L V E D V i$ left ventricular indexed end-diastolic volume, $L V E F$ left ventricular ejection fraction, $L V$ left ventricle, $R V E D V i$ right ventricular indexed end-diastolic volume, $R V E F$ right ventricular ejection fraction, $L A$ left atrium, $R A$ right atrium

Table 4 Diagnostic accuracy of CTR $=51 \%$ for detecting abnormal cardiac MRI parameters by Receiver operating characteristic analysis

\begin{tabular}{llllll}
\hline MRI parameter & AUC & Sensitivity, \% & Specificity, \% & False-negative, \% & False-positive, \% \\
\hline LVEDVi & $0.704[0.646-0.761]$ & $53.4(79 / 148)$ & $77.6(125 / 161)$ & $46.6(69 / 148)$ & $22.4(36 / 161)$ \\
LVEF & $0.669[0.607-0.731]$ & $44.6(91 / 204)$ & $77.1(81 / 105)$ & $55.4(113 / 204)$ & $22.9(24 / 105)$ \\
Indexed LVmass & $0.665[0.590-0.739]$ & $57.1(40 / 70)$ & $68.6(164 / 239)$ & $42.9(30 / 70)$ & $31.4(75 / 239)$ \\
RVEDVi & $0.719[0.640-0.798]$ & $67.9(36 / 53)$ & $69.1(177 / 256)$ & $32.1(17 / 53)$ & $30.9(79 / 256)$ \\
RVEF & $0.657[0.590-0.723]$ & $52.7(58 / 109)$ & $71.4(142 / 200)$ & $47.3(52 / 109)$ & $28.6(57 / 200)$
\end{tabular}

Values in parentheses are the numbers for percentage calculation. Values in brackets are $95 \%$ confidence intervals. $M R I$ magnetic resonance imaging, $A U C$ area under the curve, $L V E D V i$ left ventricular indexed end-diastolic volume, $L V E F$ left ventricular ejection fraction, $L V$ left ventricle, RVEDVi right ventricular indexed end-diastolic volume, RVEF right ventricular ejection fraction

\section{Correlation of CTR and cardiac MRI parameters}

THVi showed the maximum correlation with CTR values $(r=0.41)$. All other cardiac MRI parameters showed weaker correlations (Table 3, Fig. 3), which was nearly negligible in the case of RVEDVi. As expected, all parameters showed positive correlations except biventricular ejection fraction, which was negatively correlated with CTR. All correlations were statistically significant $(p<0.005)$.

\section{Comparison of patients with normal and increased CTR}

The CTR on CXR was normal in 194 cases (62.8\%) and $>50 \%$ in 115 cases $(37.2 \%)$. When grouped, patients with an increased CTR showed more abnormal cardiac MRI parameters than those with normal CTR (expressed as higher average/median ventricular volumes and atrial areas or lower ventricular ejection fraction) (Table 3). These differences were statistically significant. Nevertheless, there was a substantial overlap of individual cardiac MRI values between groups, which explains the low correlation coefficients (Figs. 3, 4).
Biventricular cardiac MRI parameters were grouped into normal and abnormal according to their age and sex reference values [15]. There was a weak but significant association $\left(r_{\phi}<0.4, p<0.05\right)$ between an increased CTR, increased indexed biventricular volumes, increased LV mass and decreased biventricular ejection fraction. ROC curves were used to represent the sensitivity and specificity of multiple CTR values graphically. A truly increased heart size was defined as increased LVEDV, LVEDVi, RVEDVi, LA area, and RA area. True-positive results (Se) and false-positive results (1-Sp) were plotted together for each variable. The diagnostic accuracy of CTR was then inferred from the area under the curve (AUC). For all variables, CTR was only mildly to moderately better than the chance to discriminate cardiac enlargement (AUC 0.6-0.7) with a large number of false positives (low $\mathrm{Sp}$ ) and false negatives (low Se), see Table 4. However, low CTR cut-off points produced very few false-negative results, and large CTR values have few false-positive results (Fig. 5, Additional file 1: Fig. S1). 


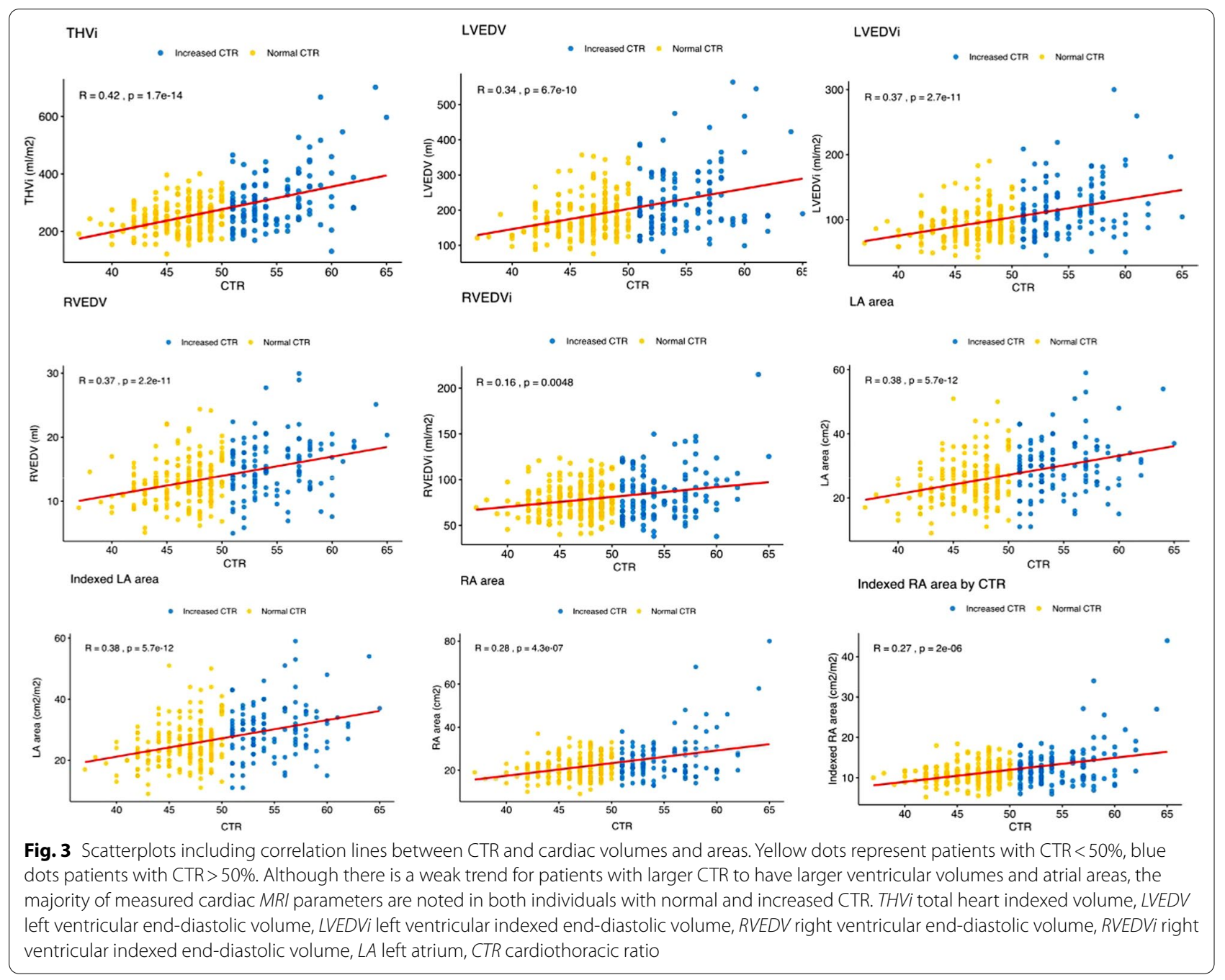

\section{Discussion}

This study aims to determine the reliability of CTR to predict cardiac enlargement by comparing the CTR values to cardiac parameters. Our results have shown a weak but statistically significant correlation between CTR and cardiac MRI defined parameters related to cardiac size. This weak correlation is explained by the substantial overlap of all measured cardiac MRI values in patients with normal and increased CTR. Although patients with CTR $>50 \%$ tend to have larger ventricular volumes and atrial areas, many patients with a normal CTR have abnormal cardiac MRI values. Interestingly, CTR correlated significantly with all ventricular and atrial measurements, despite having a broad spectrum of pathologies. In a heterogeneous adult population of our study, the cardiac chamber responsible for an increased CTR is variable, as is the number of enlarged chambers.

Compared to a paediatric population study performed by Grotenhuis et al. [2] (including congenital heart diseases, aortic regurgitation and HCM), our results show that the THVi correlated better with CTR in adults than in children $(r=0.41$ vs. $r=0.27)$. However, correlation with isolated chambers' enlargement was substantially better in children. In paediatric patients with Tetralogy of Fallot, the RVEDVi and CTR correlated weakly $(r=0.4)$, and in those with aortic regurgitation, the LVEDVi and CTR showed moderate correlation $(r=0.5)$. In this regard, a more homogenous sample is a plausible explanation for the stronger correlation between ventricular volumes and CTR, as seen in paediatric patients (i.e. enlargement of the RV is the expected cause of increased CTR in patients with Tetralogy of Fallot, as is dilated LV in patients with aortic regurgitation). On the other hand, Spiewak et al. [7] did not find a correlation between CTR and ventricular volumes in patients with Tetralogy of Fallot but reported a weak but statistically significant correlation with atrial volumes. This weak correlation is maintained in our adult population. 
Anyhow, the commented heterogeneity of results among studies is in keeping with the poor discriminatory value of CTR regarding true cardiac size, which is likely translatable in routine settings.

Notably, patients with several cardiac pathologies have been included in the present study, thus covering a broad spectrum of cardiac morphologies. Nevertheless, reliance on CTR is based on the fact that even isolated chamber enlargement impacts the whole cardiac silhouette. Typically, LV dilatation causes leftward, downward and backward rotation of the heart in a clockwise direction. However, it can also result in anticlockwise rotation, which is not expressed as increased CTR on chest radiographs $[2,16]$. Furthermore, RV does not contribute to the left heart contour unless severely dilated as the sternum limits its expansion. Only if dilated enough, RV may rotate leftwards, push the left ventricle and increase the left heart border [7]. This may help to explain the particularly low correlation found between CTR and RV volumes. As CTR effectively represents the transverse diameter between the RA and LV (which form the right and left heart borders, respectively), it might be expected to reflect more accurately the enlargement of these chambers. However, neither RA area nor LV volume showed stronger correlations with CTR than other parameters.
A large number of cases with normal CTR revealed another interesting aspect of our study. A normal CTR was determined in $62.8 \%$ of patients, even though only 8.1\% of individuals had normal cardiac MRI scans. Therefore, a CTR $<50 \%$ on chest radiographs does not seem to be a reliable indicator to exclude cardiac pathology. Dimopoulos et al. [6] also found that only $56.4 \%$ of adult congenital heart disease patients had an increased CTR on chest radiographs. In addition, some authors state that even a normal CTR could be related to poor clinical outcomes $[5,17]$. Jun et al. [17] determined that a CTR greater than $42 \%$ was an independent predictor of major adverse cardiac events after percutaneous coronary intervention. Of note, in our population, such values were rare, as only 22 individuals out of $309(7 \%)$ had a CTR of $\leq 42 \%$.

ROC curve analysis corroborated the poor discriminatory value of CTR with AUCs only mildly above "chance" when using cardiac MRI values as the gold standard. No single CTR cut-off point was able to classify increased and normal chamber sizes accurately. Low CTR thresholds were associated with many false positives (patients with higher CTR on CXR but normal heart size on cardiac MRI). Larger CTR thresholds failed to identify most patients with abnormal cardiac MRI parameters, thus

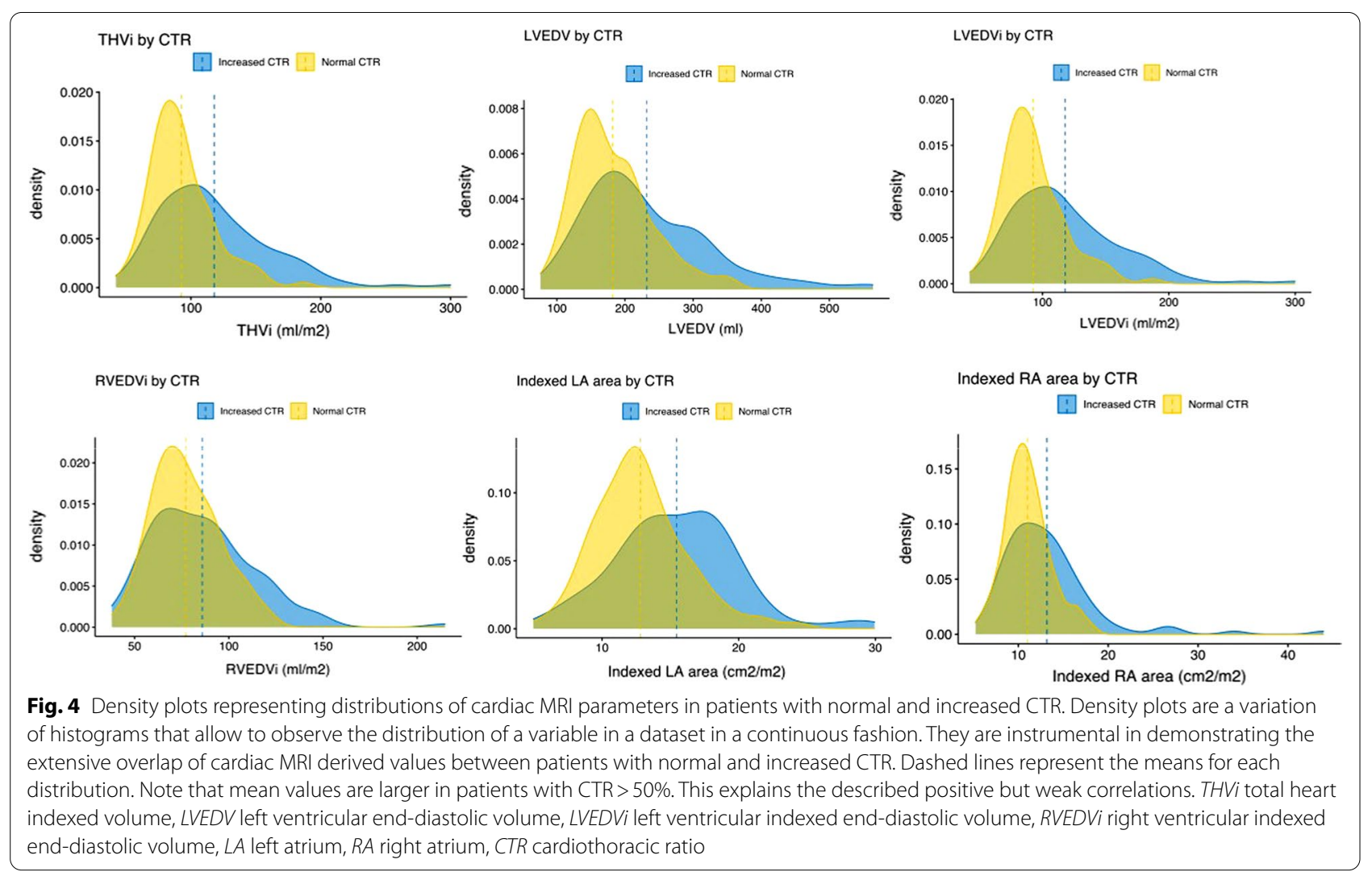




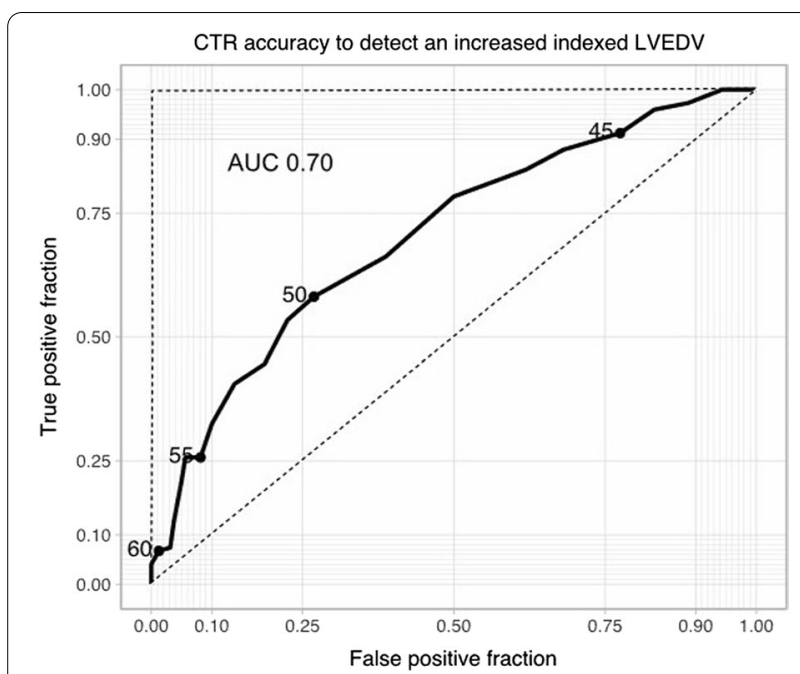

Fig. 5 ROC curve of CTR and increased LVEDV. ROC curve illustrating diagnostic accuracy of multiple CTR cut-off values to detect an increased LVEDVi. The $X$-axis shows true positives for each cut-off (an increased LVEDV on cardiac MRI is seen by the defined cut-off). The $Y$-axis shows false positives (normal LVEDV on cardiac MRI, which is characterised as increased based on a given (TR value). Higher CTR values give few false positives at the expense of many false negatives (many patients with an increased LVEDV are missed). Lower CTR values diagnose most patients with an increased LVEDV at the expense of many false-positive results. The area under the ROC curve describes the overall diagnostic power of CTR. An ideal test would have no false positives or false negatives (AUC $=1$ described by the $90^{\circ}$ dashed line). Random guessing would render $50 \%$ of true positives and $50 \%$ of false positives (AUC $=0.5$ ) and is represented by the no-discrimination line ( $45^{\circ}$ dashed diagonal line). CTR cardiothoracic ratio, LVEDVi left ventricular indexed end-diastolic volume, $A \cup C$ area under the curve. Other parameters are available in Additional file 1: Fig. S1

giving very few true positive results. Yet, CTR showed value when extreme measurements were found. That is to say, the likelihood of having an increased heart size is high when a CTR $>55 \%$ is found, as is the likelihood of having a normal heart size with a CTR $<45 \%$.

Although cardiac MRI is considered the gold standard to assess cardiac volumes and function, it is not always accessible or even indicated. Other imaging modalities such as echocardiography and $\mathrm{CT}$ are also available for cardiac evaluation. Meta-analysis performed by Loomba et al. showed that CTR was sensitive but not specific to detect left ventricular dilatation determined by echocardiography: after assessing six studies with a total of 466 patients, the authors concluded that CTR had $83.3 \%$ sensitivity, $45.4 \%$ specificity, $43.5 \%$ positive predictive value and $82.7 \%$ negative predictive value. However, after one study with a paediatric population was excluded, specificity significantly decreased to $25.2 \%$ [8]. On the contrary, CTR in patients with NSTEMI showed lower sensitivity (40\%) but higher specificity $(91 \%)$ in detecting cardiomegaly compared to echocardiography, however in this study, cardiomegaly was described as an enlargement of the right or left ventricle [18]. Furthermore, Chana et al. found CTR to have a limited value in detecting left and/or right ventricular dysfunction (0.7 AUC, 73.9\% sensitivity and $47.4 \%$ specificity) [19].

Regarding CT, CTR can be derived from the CT itself, using the scout images or axial slices. While this allows overcoming the limitation of a time gap that previous studies encountered when comparing two different imaging modalities, it is important to note that $\mathrm{CT}$ images are acquired in the supine position. Schlett et al. used anteroposterior scout images to calculate the CTR and found that CTR did not correlate with CT derived end-diastolic LV volume, mass or size (all $p \geq 0.27$ ). On the other hand, all these mentioned LV parameters showed significant correlation with a simple axial LV area-based measurement, suggesting that on $\mathrm{CT}$, other straightforward measurements may be applied instead of traditional CTR [9]. Regarding CTR as a single parameter, CTRs obtained by CXR and CT correlated $(r=0.802)$ in a study of cancer patients by Gollub et al. The authors also showed a limited ability of CT-derived CTR to recognise LV hypertrophy determined by echocardiography (AUC was 0.71) [20].

Generally, the complexity of the heart and extracardiac factors, as described in our study, undermines the value of a single cut-off of CTR. Thus, our results support the evidence that the reliability of CTR to predict cardiac size and functional status is limited.

\section{Limitations}

One of the possible limitations of our study is the heterogeneous study population. However, this allowed to assess CTR in a spectrum of pathologies in different categories, likely translatable to broader practice. Secondly, the study was performed in a specialised cardiothoracic centre, meaning there was a low proportion of healthy individuals in the study (8\%). Thirdly, our selected maximum interval between chest radiographs and cardiac MRI was one month, although the calculated average time difference was nine days. It is reasonable to assume that a time gap between studies in some cases might have been associated with clinical changes. However, in daily practice, chest radiograph and cardiac MRI are rarely performed on the same day (only $7 \%$ in our study sample). Therefore further studies assessing this may give some insight on whether the timeframe could have an influence. Thirdly, it would be interesting to evaluate separate cardiac chamber variations in the heart silhouette and whether this complements CTR to make it a more accurate measure. In this regard, artificial intelligence may play a role in automating and defining 
cardiac morphology on chest radiograph suggestive of underlying cardiac chamber abnormality. Furthermore, even though cardiac MRI is considered as the gold standard for cardiac volumetry and function, regrettably, the technique, while being increasingly used, is not yet available in every setting and is time-consuming [21]. Hence, CXR cannot be replaced by MRI, but CTR, as an estimate of true cardiac size, should be interpreted with caution and correlated with other available imaging modalities. Finally, CTR itself has limitations due to various cardiac and non-cardiac factors [3, 4]. To address this, we have constrained the impact of this limitation by excluding AP radiographs or patients with pleural or pericardial effusions obscuring the cardiac borders on chest radiographs.

\section{Conclusions}

Although CTR has an excellent interobserver agreement, is reproducible and easily measurable, it has little accuracy to distinguish a normal from an increased heart size when used as a single threshold method (i.e. CTR $>50 \%$ ). Due to the lack of sensitivity and specificity of intermediate CTR values (45-55\%), it would be more accurate to report a possibility of cardiac chamber enlargement rather than stating true cardiomegaly.

\begin{abstract}
Abbreviations
AP: Antero-posterior; CTR: Cardiothoracic ratio; CXR: Chest radiograph; EF: Ejection fraction; LA: Left atrium; LV: Left ventricle; LVEDV: Left ventricular enddiastolic volume; LVEDVi: Left ventricular indexed end-diastolic volume; LVESV: Left ventricular end-systolic volume; LVESVi: Left ventricular indexed endsystolic volume; LVOT: Left ventricular outflow tract; MRI: Magnetic resonance imaging; PA: Postero-anterior; RA: Right atrium; RV: Right ventricle; RVEDV: Right ventricular end-diastolic volume; RVEDVi: Right ventricular indexed enddiastolic volume; RVESV: Right ventricular end-systolic volume; RVESVi: Right ventricular indexed end-systolic volume; THVi: Total indexed heart volume.
\end{abstract}

\section{Supplementary Information}

The online version contains supplementary material available at https://doi. org/10.1186/s13244-021-01097-0.

Additional file 1: Fig. S1. ROC curves of CTR and: increased LVEDV, RVEDV and LV mass, decreased LVEF and RVEF. CTR cardiothoracic ratio, AUC area under the curve, LVEDV left ventricular end-diastolic volume, RVEDV right ventricular end-diastolic volume, LVEF left ventricular ejection fraction, RVEF right ventricular ejection fraction.

\section{Acknowledgements}

The authors are grateful to Professor Marc Dewey and Professor Gabriel P. Krestin for their advice and support.

\section{Authors' contributions}

All authors substantially contributed to the manuscript: Study concept and design: all authors. Literature research, material preparation and data collection: PS, MA, MG, JN, AB, DM. Data analysis: PS, MG. Manuscript preparation: PS, MG. Manuscript editing: all authors. All authors read and approved the final manuscript.

\section{Funding}

This study has received funding from the Seed Grant funding programme of the European Society of Radiology (ESR) in collaboration with the European Institute for Biomedical Imaging Research (EIBIR).

\section{Availability of data and materials}

The datasets used and/or analysed during the current study are available from the corresponding author on reasonable request.

\section{Declarations}

\section{Consent for publication}

Not applicable. All submitted images are anonymised.

\section{Competing interests}

The authors declare that they have no competing interests.

\section{Author details}

${ }^{1}$ Radiology Department, Liverpool Heart and Chest Hospital, Thomas Drive, Liverpool L14 3PE, UK. ${ }^{2}$ Department of Radiology, Lithuanian University of Health Sciences, Eiveniu 2, 50161 Kaunas, Lithuania. ${ }^{3}$ Cardiovascular Research Center-ICCC, Hospital de La Santa Creu I Sant Pau, IIB-Sant Pau, Barcelona, Spain.

Received: 2 August 2021 Accepted: 23 September 2021

Published online: 03 November 2021

\section{References}

1. Danzer CS (1919) The cardiothoracic ratio: an index of cardiac enlargement. Am J Med Sci 513-521

2. Grotenhuis HB, Zhou C, Tomlinson G et al (2015) Cardiothoracic ratio on chest radiograph in pediatric heart disease: How does it correlate with heart volumes at magnetic resonance imaging? Pediatr Radiol 45:1616-1623. https://doi.org/10.1007/s00247-015-3386-9

3. Puddy E, Hill C (2007) Interpretation of the chest radiograph. Contin Educ Anaesth Crit Care Pain 7:71-75. https://doi.org/10.1093/bjaceaccp/ mkm014

4. Kelly B (2012) The chest radiograph. Ulster Med J 81:143-148

5. Zaman MJS, Sanders J, Crook AM et al (2007) Cardiothoracic ratio within the "normal" range independently predicts mortality in patients undergoing coronary angiography. Heart 93:491-494. https://doi.org/10.1136/hrt. 2006.101238

6. Dimopoulos K, Giannakoulas G, Bendayan I et al (2013) Cardiothoracic ratio from postero-anterior chest radiographs: a simple, reproducible and independent marker of disease severity and outcome in adults with congenital heart disease. Int J Cardiol 166:453-457. https://doi.org/10. 1016/j.ijcard.2011.10.125

7. Spiewak M, Małek LA, Biernacka EK et al (2014) Cardiothoracic ratio may be misleading in the assessment of right- and left-ventricular size in patients with repaired tetralogy of Fallot. Clin Radiol 69:e1-8. https://doi. org/10.1016/j.crad.2014.03.009

8. Loomba RS, Shah PH, Nijhawan K et al (2015) Cardiothoracic ratio for prediction of left ventricular dilation: a systematic review and pooled analysis. Future Cardiol 11:171-175. https://doi.org/10.2217/fca.15.5

9. Schlett CL, Kwait DC, Mahabadi AA et al (2010) Simple area-based measurement for multidetector computed tomography to predict left ventricular size. Eur Radiol 20:1590-1596. https://doi.org/10.1007/ s00330-010-1720-z

10. Bellenger NG, Burgess MI, Ray SG et al (2000) Comparison of left ventricular ejection fraction and volumes in heart failure by echocardiography, radionuclide ventriculography and cardiovascular magnetic resonance. Are they interchangeable? Eur Heart J 21:1387-1396. https://doi.org/10. 1053/euhj.2000.2011

11. Grothues F, Smith GC, Moon JCC et al (2002) Comparison of interstudy reproducibility of cardiovascular magnetic resonance with two-dimensional echocardiography in normal subjects and in patients with heart failure or left ventricular hypertrophy. Am J Cardiol 90:29-34. https://doi. org/10.1016/s0002-9149(02)02381-0 
12. Grothues F, Moon JC, Bellenger NG et al (2004) Interstudy reproducibility of right ventricular volumes, function, and mass with cardiovascular magnetic resonance. Am Heart J 147:218-223. https://doi.org/10.1016/j. ahj.2003.10.005

13. R Core Team (2018) R: a language and environment for statistical computing. R Foundation for Statistical Computing, Vienna

14. Sachs MC (2017) plotROC: A Tool for Plotting ROC Curves. J Stat Softw Code Snippets 79:1-19. https://doi.org/10.18637/jss.v079.c02

15. Kawel-Boehm N, Maceira A, Valsangiacomo-Buechel ER et al (2015) Normal values for cardiovascular magnetic resonance in adults and children. J Cardiovasc Magn Reson. https://doi.org/10.1186/s12968-015-0111-7

16. Kono T, Suwa M, Hanada H et al (1992) Clinical significance of normal cardiac silhouette in dilated cardiomyopathy-evaluation based upon echocardiography and magnetic resonance imaging. Jpn Circ J 56:359-365. https://doi.org/10.1253/jcj.56.359

17. Jun SJ, Jeong HC, Ku YH et al (2013) An optimal cardiothoracic ratio cut-off to predict clinical outcomes in patients with acute myocardial infarction. Int J Cardiovasc Imaging 29:1889-1897. https://doi.org/10. 1007/s10554-013-0260-4

18. Is cardiomegaly on chest radiograph representative of true cardiomegaly: a cross-sectional observational study comparing cardiac size on chest radiograph to that on echocardiography. https://www.nzma.org.nz/journalarticles/is-cardiomegaly-on-chest-radiograph-representative-of-true-cardi omegaly-a-cross-sectional-observational-study-comparing-cardiac-size-onchest-radiograph-to-that-on-echocardiography. Accessed 27 Aug 2021

19. Chana HS, Martin CA, Cakebread HE et al (2015) Diagnostic accuracy of cardiothoracic ratio on admission chest radiography to detect left or right ventricular systolic dysfunction: a retrospective study. J R Soc Med 108:317-324. https://doi.org/10.1177/0141076815588314

20. Gollub MJ, Panu N, Delaney H et al (2012) Shall we report cardiomegaly at routine computed tomography of the chest? I Comput Assist Tomogr 36:67-71. https://doi.org/10.1097/RCT.0b013e318241e585

21. Mizia-Stec K, Charron P, Blanes JRG et al (2020) Availability and applicability of cardiac magnetic resonance imaging in diagnosis in cardiomyopathies: the Cardiomyopathy/Myocarditis registry of the EURObservational Research Programme of the ESC. Eur Heart J. https://doi.org/10.1093/ ehjci/ehaa946.0205

\section{Publisher's Note}

Springer Nature remains neutral with regard to jurisdictional claims in published maps and institutional affiliations.

\section{Submit your manuscript to a SpringerOpen ${ }^{\odot}$ journal and benefit from:}

- Convenient online submission

- Rigorous peer review

- Open access: articles freely available online

- High visibility within the field

- Retaining the copyright to your article

Submit your next manuscript at $\boldsymbol{\nabla}$ springeropen.com 